\title{
ADDITIVE $(\alpha, \beta)$-FUNCTIONAL EQUATIONS AND LINEAR MAPPINGS
}

\author{
CHOONKIL PARK
}

Received 18 February, 2017

Abstract. In this paper, we investigate the additive $(\alpha, \beta)$-functional equation

$$
f(x)+\bar{\alpha} f(\alpha y)+f(z)=\beta^{-1} f(\beta(x+y+z))
$$

for all complex numbers $\alpha$ with $|\alpha|=1$ and for a fixed nonzero complex number $\beta$.

Using the fixed point method and the direct method, we prove the Hyers-Ulam stability of the additive $(\alpha, \beta)$-functional equation (0.1) in complex Banach spaces.

2010 Mathematics Subject Classification: 39B52; 39B62; 47H10

Keywords: Hyers-Ulam stability, additive $(\alpha, \beta)$-functional equation, $\mathbb{C}$-linear mapping, fixed point method, direct method

\section{INTRODUCTION AND PRELIMINARIES}

The stability problem of functional equations originated from a question of Ulam [19] concerning the stability of group homomorphisms.

The functional equation $f(x+y)=f(x)+f(y)$ is called the Cauchy equation. In particular, every solution of the Cauchy equation is said to be an additive mapping. Hyers [10] gave a first affirmative partial answer to the question of Ulam for Banach spaces. Hyers' Theorem was generalized by Aoki [1] for additive mappings and by Rassias [18] for linear mappings by considering an unbounded Cauchy difference. A generalization of the Rassias theorem was obtained by Găvruta [9] by replacing the unbounded Cauchy difference by a general control function in the spirit of Rassias' approach. See $[2,6,8,12,15,16,20]$ for more information on functional equations.

We recall a fundamental result in fixed point theory.

Theorem $1([3,7])$. Let $(X, d)$ be a complete generalized metric space and let $J: X \rightarrow X$ be a strictly contractive mapping with Lipschitz constant $\alpha<1$. Then for

This work was supported by Basic Science Research Program through the National Research Foundation of Korea funded by the Ministry of Education, Science and Technology (NRF2017R1D1A1B04032937). 
each given element $x \in X$, either

$$
d\left(J^{n} x, J^{n+1} x\right)=\infty
$$

for all nonnegative integers $n$ or there exists a positive integer $n_{0}$ such that

(1) $d\left(J^{n} x, J^{n+1} x\right)<\infty, \quad \forall n \geq n_{0}$;

(2) the sequence $\left\{J^{n} x\right\}$ converges to a fixed point $y^{*}$ of $J$;

(3) $y^{*}$ is the unique fixed point of $J$ in the set $Y=\left\{y \in X \mid d\left(J^{n_{0}} x, y\right)<\infty\right\}$;

(4) $d\left(y, y^{*}\right) \leq \frac{1}{1-\alpha} d(y, J y)$ for all $y \in Y$.

In 1996, G. Isac and Th.M. Rassias [11] were the first to provide applications of stability theory of functional equations for the proof of new fixed point theorems with applications. By using fixed point methods, the stability problems of several functional equations have been extensively investigated by a number of authors (see $[4,5,17])$.

In Section 2, we solve the additive $(\alpha, \beta)$-functional equation ( 0.1$)$ in vector spaces and prove the Hyers-Ulam stability of the additive $(\alpha, \beta)$-functional equation $(0.1)$ in Banach spaces by using the fixed point method.

In Section 3, we prove the Hyers-Ulam stability of the additive $(\alpha, \beta)$-functional equation (0.1) in Banach spaces by using the direct method.

Throughout this paper, assume that $X$ is a complex normed space and that $Y$ is a complex Banach space. Let $\beta$ be a fixed nonzero complex number.

\section{AdDitive $(\alpha, \beta)$-FUnCTIONAL EQUATION (0.1) IN COMPLEX BANACH SPACES $I$}

We solve the additive $(\alpha, \beta)$-functional equation $(0.1)$ in complex vector spaces.

Lemma 1. Let $X$ and $Y$ be complex vector spaces. If a mapping $f: X \rightarrow Y$ satisfies

$$
f(x)+\bar{\alpha} f(\alpha y)+f(z)=\beta^{-1} f(\beta(x+y+z))
$$

for all $x, y, z \in X$ and all $\alpha \in \mathbb{T}:=\{\mu \in \mathbb{C}|| \mu \mid=1\}$, then $f: X \rightarrow Y$ is $\mathbb{C}$-linear.

Proof. Assume that $f: X \rightarrow Y$ satisfies (2.1).

Letting $x=y=z=0$ in (2.1), we get $(2+\bar{\alpha}) f(0)=\beta^{-1} f(0)$ for all $\alpha \in \mathbb{T}$. So $f(0)=0$.

Letting $\alpha=1, y=-x$ and $z=0$ in (2.1), we get $f(x)+f(-x)=0$ and so $f(-x)=-f(x)$ for all $x \in X$.

Letting $\alpha=1$ and $z=-x-y$ in (2.1), we get

$$
f(x)+f(y)-f(x+y)=f(x)+f(y)+f(-x-y)=0
$$

and so

$$
f(x+y)=f(x)+f(y)
$$

for all $x, y \in X$. 
Letting $z=0$ and $y=-x$ in (2.1), we get $f(x)+\bar{\alpha} f(-\alpha x)=0$ and so $f(\alpha x)=$ $\alpha f(x)$ for all $x \in X$ and all $\alpha \in \mathbb{T}$. By the same reasoning as in the proof of [14, Theorem 2.1], the mapping $f: X \rightarrow Y$ is $\mathbb{C}$-linear.

Using the fixed point method, we prove the Hyers-Ulam stability of the additive $(\alpha, \beta)$-functional equation (2.1) in complex Banach spaces.

Theorem 2. Let $\varphi: X^{3} \rightarrow[0, \infty)$ be a function such that there exists an $L<1$ with

$$
\varphi\left(\frac{x}{2}, \frac{y}{2}, \frac{z}{2}\right) \leq \frac{L}{2} \varphi(x, y, z)
$$

for all $x, y, z \in X$. Let $f: X \rightarrow Y$ be a mapping satisfying $f(0)=0$ and

$$
\left\|f(x)+\bar{\alpha} f(\alpha y)+f(z)-\beta^{-1} f(\beta(x+y+z))\right\| \leq \varphi(x, y, z)
$$

for all $x, y, z \in X$ and all $\alpha \in \mathbb{T}$. Then there exists a unique $\mathbb{C}$-linear mapping $A: X \rightarrow Y$ such that

$$
\|f(x)-A(x)\| \leq \frac{L}{2(1-L)}(\varphi(-x,-x, 2 x)+2 \varphi(-x, x, 0))
$$

for all $x \in X$.

Proof. Let $\alpha=1$.

Replacing $x$ by $-x$ and letting $y=-x$ and $z=2 x$ in (2.3), we get

$$
\|2 f(-x)+f(2 x)\| \leq \varphi(-x,-x, 2 x)
$$

for all $x \in X$.

Replacing $x$ by $-x$ and letting $y=x$ and $z=0$ in (2.3), we get

$$
\|f(-x)+f(x)\| \leq \varphi(-x, x, 0)
$$

for all $x \in X$.

It follows from (2.5) and (2.6) that

$$
\|f(2 x)-2 f(x)\| \leq \varphi(-x,-x, 2 x)+2 \varphi(-x, x, 0)
$$

for all $x \in X$.

Consider the set

$$
S:=\{h: X \rightarrow Y, h(0)=0\}
$$

and introduce the generalized metric on $S$ :

$$
\begin{aligned}
& d(g, h) \\
& =\inf \left\{\mu \in \mathbb{R}_{+}:\|g(x)-h(x)\| \leq \mu(\varphi(-x,-x, 2 x)+2 \varphi(-x, x, 0)), \quad \forall x \in X\right\},
\end{aligned}
$$

where, as usual, $\inf \phi=+\infty$. It is easy to show that $(S, d)$ is complete (see [13]).

Now we consider the linear mapping $J: S \rightarrow S$ such that

$$
J g(x):=2 g\left(\frac{x}{2}\right)
$$


for all $x \in X$.

Let $g, h \in S$ be given such that $d(g, h)=\varepsilon$. Then

$$
\|g(x)-h(x)\| \leq \varepsilon(\varphi(-x,-x, 2 x)+2 \varphi(-x, x, 0))
$$

for all $x \in X$. Hence

$$
\begin{aligned}
\|J g(x)-J h(x)\| & =\left\|2 g\left(\frac{x}{2}\right)-2 h\left(\frac{x}{2}\right)\right\| \\
& \leq 2 \varepsilon\left(\varphi\left(-\frac{x}{2},-\frac{x}{2}, x\right)+2 \varphi\left(-\frac{x}{2}, \frac{x}{2}, 0\right)\right) \\
& \leq 2 \varepsilon \frac{L}{2}(\varphi(-x,-x, 2 x)+2 \varphi(-x, x, 0)) \\
& \leq L \varepsilon(\varphi(-x,-x, 2 x)+2 \varphi(-x, x, 0))
\end{aligned}
$$

for all $x \in X$. So $d(g, h)=\varepsilon$ implies that $d(J g, J h) \leq L \varepsilon$. This means that

$$
d(J g, J h) \leq L d(g, h)
$$

for all $g, h \in S$.

It follows from (2.7) that

$$
\begin{aligned}
\left\|f(x)-2 f\left(\frac{x}{2}\right)\right\| & \leq \varphi\left(-\frac{x}{2},-\frac{x}{2}, x\right)+2 \varphi\left(-\frac{x}{2}, \frac{x}{2}, 0\right) \\
& \leq \frac{L}{2}(\varphi(-x,-x, 2 x)+2 \varphi(-x, x, 0))
\end{aligned}
$$

for all $x \in X$. So $d(f, J f) \leq \frac{L}{2}$.

By Theorem 1, there exists a mapping $A: X \rightarrow Y$ satisfying the following:

(1) $A$ is a fixed point of $J$, i.e.,

$$
A(x)=2 A\left(\frac{x}{2}\right)
$$

for all $x \in X$. The mapping $A$ is a unique fixed point of $J$ in the set

$$
M=\{g \in S: d(f, g)<\infty\} .
$$

This implies that $A$ is a unique mapping satisfying (2.8) such that there exists a $\mu \in$ $(0, \infty)$ satisfying

$$
\|f(x)-A(x)\| \leq \mu(\varphi(-x,-x, 2 x)+2 \varphi(-x, x, 0))
$$

for all $x \in X$;

(2) $d\left(J^{l} f, A\right) \rightarrow 0$ as $l \rightarrow \infty$. This implies the equality

$$
\lim _{l \rightarrow \infty} 2^{n} f\left(\frac{x}{2^{n}}\right)=A(x)
$$

for all $x \in X$;

(3) $d(f, A) \leq \frac{1}{1-L} d(f, J f)$, which implies

$$
\|f(x)-A(x)\| \leq \frac{L}{2(1-L)}(\varphi(-x,-x, 2 x)+2 \varphi(-x, x, 0))
$$


for all $x \in X$.

It follows from (2.2) and (2.3) that

$$
\begin{aligned}
& \left\|A(x)+\bar{\alpha} A(\alpha y)+A(z)-\beta^{-1} A(\beta(x+y+z))\right\| \\
& =\lim _{n \rightarrow \infty} 2^{n}\left\|f\left(\frac{x}{2^{n}}\right)+\bar{\alpha} f\left(\frac{\alpha y}{2^{n}}\right)+f\left(\frac{z}{2^{n}}\right)-\beta^{-1} f\left(\beta\left(\frac{x+y+z}{2^{n}}\right)\right)\right\| \\
& \leq \lim _{n \rightarrow \infty} 2^{n} \varphi\left(\frac{x}{2^{n}}, \frac{y}{2^{n}}, \frac{z}{2^{n}}\right)=0
\end{aligned}
$$

for all $x, y, z \in X$ and all $\alpha \in \mathbb{T}$. So

$$
A(x)+\bar{\alpha} A(\alpha y)+A(z)-\beta^{-1} A(\beta(x+y+z))=0
$$

for all $x, y, z \in X$ and all $\alpha \in \mathbb{T}$. By Lemma 1 , the mapping $A: X \rightarrow Y$ is $\mathbb{C}$ linear.

Corollary 1. Let $r>1$ and $\theta$ be nonnegative real numbers, and let $f: X \rightarrow Y$ be a mapping satisfying

$$
\left\|f(x)+\bar{\alpha} f(\alpha y)+f(z)-\beta^{-1} f(\beta(x+y+z))\right\| \leq \theta\left(\|x\|^{r}+\|y\|^{r}+\|z\|^{r}\right)
$$

for all $x, y, z \in X$ and all $\alpha \in \mathbb{T}$. Then there exists a unique $\mathbb{C}$-linear mapping $A: X \rightarrow Y$ such that

for all $x \in X$.

$$
\|f(x)-A(x)\| \leq \frac{2^{r}+6}{2^{r}-2} \theta\|x\|^{r}
$$

Proof. The proof follows from Theorem 2 by taking $\varphi(x, y, z)=\theta\left(\|x\|^{r}+\|y\|^{r}+\right.$ $\left.\|z\|^{r}\right)$ for all $x, y, z \in X$. Then we can choose $L=2^{1-r}$ and we get the desired result.

Theorem 3. Let $\varphi: X^{3} \rightarrow[0, \infty)$ be a function such that there exists an $L<1$ with

$$
\varphi(x, y, z) \leq 2 L \varphi\left(\frac{x}{2}, \frac{y}{2}, \frac{z}{2}\right)
$$

for all $x, y, z \in X$. Let $f: X \rightarrow Y$ be a mapping satisfying $f(0)=0$ and (2.3). Then there exists a unique $\mathbb{C}$-linear mapping $A: X \rightarrow Y$ such that

$$
\|f(x)-A(x)\| \leq \frac{1}{2(1-L)}(\varphi(-x,-x, 2 x)+2 \varphi(-x, x, 0))
$$

for all $x \in X$.

Proof. It follows from (2.7) that

$$
\left\|f(x)-\frac{1}{2} f(2 x)\right\| \leq \frac{1}{2}(\varphi(-x,-x, 2 x)+2 \varphi(-x, x, 0))
$$

for all $x \in X$.

Let $(S, d)$ be the generalized metric space defined in the proof of Theorem 2 . 
Now we consider the linear mapping $J: S \rightarrow S$ such that

$$
J g(x):=\frac{1}{2} g(2 x)
$$

for all $x \in X$.

The rest of the proof is similar to the proof of Theorem 2 .

Corollary 2. Let $r<1$ and $\theta$ be positive real numbers, and let $f: X \rightarrow Y$ be a mapping satisfying (2.9). Then there exists a unique $\mathbb{C}$-linear mapping $A: X \rightarrow Y$ such that

for all $x \in X$.

$$
\|f(x)-A(x)\| \leq \frac{6+2^{r}}{2-2^{r}} \theta\|x\|^{r}
$$

Proof. The proof follows from Theorem 3 by taking $\varphi(x, y, z)=\theta\left(\|x\|^{r}+\|y\|^{r}+\right.$ $\left.\|z\|^{r}\right)$ for all $x, y, z \in X$. Then we can choose $L=2^{r-1}$ and we get desired result.

\section{Additive $(\alpha, \beta)$-FUnCTIONAL EQUATION (0.1) IN COMPLEX BANACH}

$$
\text { SPACES } I I
$$

In this section, using the direct method, we prove the Hyers-Ulam stability of the additive $(\alpha, \beta)$-functional equation (2.1) in complex Banach spaces.

Theorem 4. Let $\varphi: X^{3} \rightarrow[0, \infty)$ be a function and let $f: X \rightarrow Y$ be a mapping satisfying $f(0)=0$ and

$$
\begin{gathered}
\Psi(x, y, z):=\sum_{j=1}^{\infty} 2^{j} \varphi\left(\frac{x}{2^{j}}, \frac{y}{2^{j}}, \frac{z}{2^{j}}\right)<\infty, \\
\left\|f(x)+\bar{\alpha} f(\alpha y)+f(z)-\beta^{-1} f(\beta(x+y+z))\right\| \leq \varphi(x, y, z)
\end{gathered}
$$

for all $x, y, z \in X$ and all $\alpha \in \mathbb{T}$. Then there exists a unique $\mathbb{C}$-linear mapping $A: X \rightarrow Y$ such that

$$
\|f(x)-A(x)\| \leq \frac{1}{2}(\Psi(-x,-x, 2 x)+2 \Psi(-x, x, 0))
$$

for all $x \in X$.

Proof. Let $\alpha=1$.

It follows from (2.7) that

$$
\left\|f(x)-2 f\left(\frac{x}{2}\right)\right\| \leq \varphi\left(-\frac{x}{2},-\frac{x}{2}, x\right)+2 \varphi\left(-\frac{x}{2}, \frac{x}{2}, 0\right)
$$

for all $x \in X$. Hence

$$
\left\|2^{l} f\left(\frac{x}{2^{l}}\right)-2^{m} f\left(\frac{x}{2^{m}}\right)\right\| \leq \sum_{j=l}^{m-1}\left\|2^{j} f\left(\frac{x}{2^{j}}\right)-2^{j+1} f\left(\frac{x}{2^{j+1}}\right)\right\|
$$




$$
\leq \sum_{j=l}^{m-1}\left(2^{j} \varphi\left(-\frac{x}{2^{j+1}},-\frac{x}{2^{j+1}}, \frac{x}{2^{j}}\right)+2^{j+1} \varphi\left(-\frac{x}{2^{j+1}}, \frac{x}{2^{j+1}}, 0\right)\right)
$$

for all nonnegative integers $m$ and $l$ with $m>l$ and all $x \in X$. It follows from (3.3) that the sequence $\left\{2^{k} f\left(\frac{x}{2^{k}}\right)\right\}$ is Cauchy for all $x \in X$. Since $Y$ is a Banach space, the sequence $\left\{2^{k} f\left(\frac{x}{2^{k}}\right)\right\}$ converges. So one can define the mapping $A: X \rightarrow Y$ by

$$
A(x):=\lim _{k \rightarrow \infty} 2^{k} f\left(\frac{x}{2^{k}}\right)
$$

for all $x \in X$. Moreover, letting $l=0$ and passing the limit $m \rightarrow \infty$ in (3.3), we get (3.2).

Now, let $T: X \rightarrow Y$ be another additive mapping satisfying (3.2). Then we have

$$
\begin{aligned}
\|A(x)-T(x)\| & =\left\|2^{q} A\left(\frac{x}{2^{q}}\right)-2^{q} T\left(\frac{x}{2^{q}}\right)\right\| \\
& \leq\left\|2^{q} A\left(\frac{x}{2^{q}}\right)-2^{q} f\left(\frac{x}{2^{q}}\right)\right\|+\left\|2^{q} T\left(\frac{x}{2^{q}}\right)-2^{q} f\left(\frac{x}{2^{q}}\right)\right\| \\
& \leq 2^{q} \Psi\left(-\frac{x}{2^{q}},-\frac{x}{2^{q}}, \frac{2 x}{2^{q}}\right)+2^{q+1} \Psi\left(-\frac{x}{2^{q}}, \frac{x}{2^{q}}, 0\right),
\end{aligned}
$$

which tends to zero as $q \rightarrow \infty$ for all $x \in X$. So we can conclude that $A(x)=T(x)$ for all $x \in X$. This proves the uniqueness of $A$.

The rest of the proof is similar to the proof of Theorem 2.

Corollary 3. Let $r>1$ and $\theta$ be nonnegative real numbers, and let $f: X \rightarrow Y$ be a mapping satisfying (2.9). Then there exists a unique $\mathbb{C}$-linear mapping $A: X \rightarrow Y$ such that

for all $x \in X$.

$$
\|f(x)-A(x)\| \leq \frac{2^{r}+6}{2^{r}-2} \theta\|x\|^{r}
$$

Proof. The proof follows from Theorem 4 by taking $\varphi(x, y, z)=\theta\left(\|x\|^{r}+\|y\|^{r}+\right.$ $\|z\|^{r}$ ) for all $x, y, z \in X$.

Theorem 5. Let $\varphi: X^{3} \rightarrow[0, \infty)$ be a function and let $f: X \rightarrow Y$ be a mapping satisfying $f(0)=0,(3.1)$ and

$$
\Psi(x, y, z):=\sum_{j=0}^{\infty} \frac{1}{2^{j}} \varphi\left(2^{j} x, 2^{j} y, 2^{j} z\right)<\infty
$$

for all $x, y, z \in X$. Then there exists a unique $\mathbb{C}$-linear mapping $A: X \rightarrow Y$ such that

$$
\|f(x)-A(x)\| \leq \frac{1}{2}(\Psi(-x,-x, 2 x)+2 \Psi(-x, x, 0))
$$

for all $x \in X$. 
Proof. It follows from (2.7) that

$$
\left\|f(x)-\frac{1}{2} f(2 x)\right\| \leq(\varphi(-x,-x, 2 x)+2 \varphi(-x, x, 0))
$$

for all $x \in X$. Hence

$$
\begin{gathered}
\left\|\frac{1}{2^{l}} f\left(2^{l} x\right)-\frac{1}{2^{m}} f\left(2^{m} x\right)\right\| \leq \sum_{j=l}^{m-1}\left\|\frac{1}{2^{j}} f\left(2^{j} x\right)-\frac{1}{2^{j+1}} f\left(2^{j+1} x\right)\right\| \\
\leq \sum_{j=l}^{m-1}\left(\frac{1}{2^{j+1}} \varphi\left(-2^{j} x,-2^{j} x, 2^{j+1} x\right)+\frac{1}{2^{j}} \varphi\left(-2^{j} x, 2^{j} x, 0\right)\right)
\end{gathered}
$$

for all nonnegative integers $m$ and $l$ with $m>l$ and all $x \in X$. It follows from (3.5) that the sequence $\left\{\frac{1}{2^{n}} f\left(2^{n} x\right)\right\}$ is a Cauchy sequence for all $x \in X$. Since $Y$ is complete, the sequence $\left\{\frac{1}{2^{n}} f\left(2^{n} x\right)\right\}$ converges. So one can define the mapping $A: X \rightarrow Y$ by

$$
A(x):=\lim _{n \rightarrow \infty} \frac{1}{2^{n}} f\left(2^{n} x\right)
$$

for all $x \in X$. Moreover, letting $l=0$ and passing the limit $m \rightarrow \infty$ in (3.5), we get (3.4).

The rest of the proof is similar to the proofs of Theorems 2 and 4 .

Corollary 4. Let $r<1$ and $\theta$ be positive real numbers, and let $f: X \rightarrow Y$ be a mapping satisfying (2.9). Then there exists a unique $\mathbb{C}$-linear mapping $A: X \rightarrow Y$ such that

for all $x \in X$.

$$
\|f(x)-A(x)\| \leq \frac{6+2^{r}}{2-2^{r}} \theta\|x\|^{r}
$$

Proof. The proof follows from Theorem 5 by taking $\varphi(x, y, z)=\theta\left(\|x\|^{r}+\|y\|^{r}+\right.$ $\left.\|z\|^{r}\right)$ for all $x, y, z \in X$.

\section{REFERENCES}

[1] T. Aoki, "On the stability of the linear transformation in Banach spaces," J. Math. Soc. Japan, vol. 2, pp. 64-66, 1950, doi: 10.2969/jmsj/00210064.

[2] A. Chahbi and N. Bounader, "On the generalized stability of d'Alembert functional equation," $J$. Nonlinear Sci. Appl., vol. 6, pp. 198-204, 2013.

[3] L. Cădariu and V. Radu, "Fixed points and the stability of Jensen's functional equation," J. Inequal. Pure Appl. Math., vol. 4, no. 1, pp. 1-4, 2003.

[4] L. Cădariu and V. Radu, "On the stability of the Cauchy functional equation: a fixed point approach,” Grazer Math. Ber., vol. 346, pp. 43-52, 2004.

[5] L. Cădariu and V. Radu, "Fixed point methods for the generalized stability of functional equations in a single variable," Fixed Point Theory Appl., vol. 2008, pp. 1-13, 2008, doi: $10.1155 / 2008 / 749392$. 
[6] A. C. D. Zeglami and S. Kabba, "On the superstability of the Pexider type generalized trigonometric functional equations," Acta Math. Sci. Ser. B Engl. Ed., vol. 34, pp. 1749-1760, 2014, doi: 10.1016/S0252-9602(14)60120-X.

[7] J. Diaz and B. Margolis, "A fixed point theorem of the alternative for contractions on a generalized complete metric space," Bull. Am. Math. Soc., vol. 74, pp. 305-309, 1968.

[8] G. Z. Eskandani and P. Găvruta, "Hyers-Ulam-Rassias stability of pexiderized Cauchy functional equation in 2-Banach spaces," J. Nonlinear Sci. Appl., vol. 5, pp. 459-465, 2012.

[9] P. Găvruta, "A generalization of the Hyers-Ulam-Rassias stability of approximately additive mappings," J. Math. Anal. Appl., vol. 184, pp. 431-433, 1994.

[10] D. Hyers, "On the stability of the linear functional equation," Proc. Natl. Acad. Sci. U.S.A., vol. 27 , pp. 222-224, 1941.

[11] G. Isac and T. M. Rassias, "Stability of $\psi$-additive mappings: Applications to nonlinear analysis," Int. J. Math. Math. Sci., vol. 19, pp. 219-228, 1996.

[12] E. T. K. Ravi and B. Senthil Kumar, "Solution and stability of a reciprocal type functional equation in several variables," J. Nonlinear Sci. Appl., vol. 7, pp. 18-27, 2014.

[13] D. Miheț and V. Radu, "On the stability of the additive Cauchy functional equation in random normed spaces," J. Math. Anal. Appl., vol. 343, pp. 567-572, 2008, doi: 10.1016/j.jmaa.2008.01.100.

[14] C. Park, "Homomorphisms between Poisson $J C^{*}$-algebras," Bull. Braz. Math. Soc., vol. 36, pp. 79-97, 2005.

[15] C. Park, "Orthogonal stability of a cubic-quartic functional equation," J. Nonlinear Sci. Appl., vol. 5, pp. 28-36, 2012.

[16] C. Park, "Quadratic $\rho$-functional inequalities in Banach spaces," Acta Math. Sci. Ser. B Engl. Ed., vol. 35, pp. 1501-1510, 2015, doi: 10.1016/S0252-9602(15)30071-0.

[17] V. Radu, "The fixed point alternative and the stability of functional equations," Fixed Point Theory, vol. 4, pp. 91-96, 2003.

[18] T. Rassias, "On the stability of the linear mapping in Banach spaces," Proc. Am. Math. Soc., vol. 72, pp. 297-300, 1978.

[19] S. Ulam, A Collection of the Mathematical Problems. New York: Interscience Publ., 1960.

[20] C. Zaharia, "On the probabilistic stability of the monomial functional equation," J. Nonlinear Sci. Appl., vol. 6, pp. 51-59, 2013.

Author's address

Choonkil Park

Hanyang University, Department of Department, Research Institute for Natural Sciences, Seoul 04763, Republic of Korea

E-mail address: baak@hanyang.ac.kr 